\title{
Design von Produkt-Dienstleistungssystemen für Kreislaufwirtschaft
}

Ursula Tischner

Die Kreislaufwirtschaft wird von den verschiedensten Seiten als wichtige Strategie für eine nachhaltige Zukunft gefordert und gefördert. Schon seit einiger Zeit setzt sich die Erkenntnis durch, dass eine Bewegung weg von Geschäftsmodellen, die auf Produktverkäufen basieren, hin zum Angebot von Produkt-Dienstleistungssystemen das Implementieren von Kreislaufwirtschaft unterstützen kann. Diese These wurde erneut in der Arbeit der Circular Economy Initiative Deutschland (CEID) diskutiert und in Berichten dieser Initiative dokumentiert. Die Autorin, die auch Mitglied der Circular Economy Initiative war, beleuchtet in diesem Text den Zusammenhang und beschreibt anhand von Ergebnissen aus früheren Forschungs- und Beratungstätigkeiten, wie Produkt-Dienstleistungssysteme (Product-Service Systems, PSS) die Circular Economy unterstützen können und wie diese PSS gestaltet werden können, damit das möglichst nachhaltig, also ökologisch, sozial und ökonomisch vorteilhaft, wirkt. Prozesse, Methoden und Tools für PSS Design und Evaluierung werden vorgestellt und durch Fallbeispiele illustriert.

Keywords: Kreislaufwirtschaft, Produkt-Dienstleistungssysteme, Circular Economy, Product-Service Systems, nachhaltiges Design

\section{Einleitung: Kreislaufwirtschaft}

Die bisher vorherrschende Produktions- und Konsumweise des „Take, Make, Waste" stößt zunehmend an planetare Belastungsgrenzen. Die Menschheit verbraucht im globalen Durchschnitt momentan die natürlichen Ressourcen von 1,6 Erden. Deutschland mit seinem Jahresverbrauch von ungefähr drei Erden steht dabei im Mittelfeld (Earth Overshoot Day 2020). Die meisten Länder dieser Welt verbrauchen also mehr und schneller Ressourcen, als der Planet Erde erneuern kann. Die globale Ressourcennutzung verursacht 50 Prozent der globalen Treibhausgas-Emissionen und 90 Prozent des Biodiversitätsverlusts und des Wasserstresses (International Resource Panel 2019). Wenn für zukünftige Generationen ein würdevolles Leben auf der Erde möglich sein soll, dann muss diese destruktive Art zu Wirtschaften aufhören.

Das Konzept der Circular Economy oder Kreislaufwirtschaft kann dazu beitragen, durch Kreislaufschließung die Nutzung von Ressourcen vom Wirtschaftswachstum abzukoppeln (vgl. acatech et al. 2020). Während derzeit eine relative Entkopplung auf Basis vorgenommener Effizienzsteigerungen teilweise schon gelingt, bedarf es in Zukunft 
jedoch einer absoluten Entkopplung, um bei steigendem Wohlstandniveau insbesondere der ärmeren Bevölkerungsschichten geringere Ressourcenverbräuche zu realisieren. Ergebnisse des International Resource Panel deuten darauf hin, dass Kreislaufwirtschaftsstrategien in der Gruppe der G7-Länder im Jahr 2050 auch die Treibhausgasemissionen von Gebäuden und Fahrzeugen um bis zu je 40 \% senken könnten (International Resource Panel 2020). Die europäische Industrie könnte durch Maßnahmen wie Materialeffizienz, Wiederverwendung und Rohstoffrückgewinnung bei der Nutzung ihrer vier emissionsstärksten Materialien (Stahl, Kunststoff, Aluminium und Zement), im Jahr 2050 bis zu 60 \% Treibhausgaseinsparungen erzielen im Vergleich zum „business as usual“ Szenario (Material Economics 2018).

Ganz im Sinne des Re-Think, Reduce, Reuse, Recycle (Schlagworte der Circular Economy) beinhaltet die Kreislaufwirtschaft sowohl Effizienzstrategien als auch Suffizienzund Konsistenzansätze. Sie verbindet somit Klima- und Ressourcenschutz, Steigerung der Wettbewerbsfähigkeit und Rohstoffunabhängigkeit sowie Schaffung von Beschäftigung und lokaler Wertschöpfung und kann damit nachhaltige win-win-win Lösungen hervorbringen (acatech et al. 2020).

\section{Eine kurze Geschichte: Kreislaufwirtschaft und Product-Service Systems}

Die Idee der Kreislaufwirtschaft ist nicht neu. Im Jahr 1976 verabschiedete der US-Kongress den "Resource Conservation and Recovery Act" (US Congress 1976) zur Förderung von Abfallvermeidung, Recycling und Ressourcenschonung. So entstanden die 3 Rs (Reduce, Reuse, Recycle) als Slogan, um die Idee der Bevölkerung zu vermitteln. Deutschland verabschiedete 1996 ein Kreislaufwirtschaft und Abfallgesetz, das in 2012 überarbeitet und aktualisiert wurde (KrW-/AbfG 2012). Beide Gesetze entstanden im Rahmen von Abfallvermeidungsansätzen, gehen das Thema also vom Abfall her an.

Re-Think Ansätze, die Kreislaufwirtschaftsstrategien bereits in die Entstehung von Produkten und Geschäftsmodellen integrieren entwickelte Walter Stahel vom Institut für Produktdauerforschung in der Schweiz bereits in den 1980er Jahren. Er prägte den Begriff der "Performance Economy“ (Stahel 2010). Ende der 1990er-Jahre wurde das Cradle to Cradle Design Prinzip vom deutschen Chemiker Michael Braungart und dem US-amerikanischen Architekten William McDonough formuliert. Sie schlugen vor, Produkte so zu gestalten, dass Materialien in natürlichen oder technischen Kreisläufen geführt werden können und so Reststoffe zu Nährstoffen werden (Braungart/ McDonough 2014). Das Planen und Gestalten von Kreislaufstrategien bereits in der 
Produktdesign und -entwicklungsphase ist auch ein fester Bestandteil vom ökologischen oder Eco-Design, wie es in den frühen 1990er Jahren von der Autorin definiert und formuliert wurde (Schmidt-Bleek/Tischner 1995). In diesem Zusammenhang entstand auch die folgende Klassifizierung von Circular Design Strategien (basierend auf der VDI 2243):

Tabelle 1: Circular Design Strategien aus den 1990er Jahren

\begin{tabular}{ll}
\hline Upcycling/Aufwertung: & $\begin{array}{l}\text { Veredelungsprozesse, bei denen ausrangierte Produkte oder } \\
\text { Materialien durch Aufwertung neue hochwertigere Funktionen } \\
\text { erhalten (alte LKW-Planen werden zu Taschen) }\end{array}$ \\
\hline Wiederverwendung: & $\begin{array}{l}\text { bereits gebrauchte Produkte werden für denselben Zweck er- } \\
\text { neut verwendet (Mehrwegverpackungen) }\end{array}$ \\
\hline Weiterverwendung: & $\begin{array}{l}\text { bereits gebrauchte Produkte werden für einen anderen als den } \\
\text { nem Trinkglas) }\end{array}$ \\
\hline Wiederverwertung: & $\begin{array}{l}\text { erneuter Einsatz von Altstoffen und Produktionsabfällen in ei- } \\
\text { nem gleichartigen Produktionsprozess (Umschmelzen von Glas) }\end{array}$ \\
\hline \multirow{2}{*}{ Weiterverwertung: } & $\begin{array}{l}\text { Einsatz von Altstoffen und Produktionsabfällen in einem von } \\
\text { diesen noch nicht durchlaufenen Produktionsprozess, wodurch } \\
\text { andere Werkstoffe oder Produkte mit anderen Eigenschaften } \\
\text { entstehen }\end{array}$
\end{tabular}

In Produktentwicklung und Design können solche Strategien bereits frühzeitig antizipiert und das Design dementsprechend detailliert werden durch zerlegungsfreundliche Gestaltung, Einsatz von Rezyklaten und gebrauchten Komponenten, Verwendung von wieder-/weiterverwendbaren Komponenten und wieder-/weiterverwertbaren Materialien etc.

Bald setzte sich jedoch die Erkenntnis durch, dass solche Kreislaufstrategien, selbst wenn sie im Produktdesign angelegt waren, in der Realität nicht immer realisiert werden konnten, da die Nutzer:innen sich nicht so verhielten wie erwartet, oder die Infrastruktur in der Praxis nicht zur Verfügung stand, das Geschäftsmodell nicht unterstütze usw. Diese Hemmnisse und Rebound-Effekte, führten zu der Annahme, dass Circular Design besser funktionieren würde, wenn nicht nur ein Produkt, sondern das gesamte System um die Nutzung herum, der Context of Use, mitgestaltet würde. Warum sollte ein zirkulär gestaltetes Produkt überhaupt noch verkauft werden? Die Anbieter hätten doch ein Interesse die Produkte, Komponenten und Materialien unter 
Kontrolle zu behalten und im richtigen, effizientesten und profitabelsten Moment wieder in den Kreislauf einzubringen. So wurde die Kombination von Produkt-Dienstleistungssystemen (Product-Service Systems, PSS, auch eco-efficient Services, Servicizing, Product as a Service, PaaS, Systeminnovationen etc.) mit der Kreislaufwirtschaft auch für Designer:innen interessant. Das Thema der Nutzungsdauer von Produkten wird bereits seit Ende der siebziger Jahre bearbeitet (vgl. Lund 1977; OECD 1982). Zentrale Beiträge zur Konzeptionierung und praktischen Umsetzung von Konzepten zur längeren und intensiveren Güternutzung, die bis hin zu Visionen einer "Verdienstleistung" heutiger Wirtschaftssysteme reichen, gingen wiederum von Stahel aus (z.B. Stahel 1991). Aus Nachhaltigkeitssicht sind Produkt-Dienstleistungssysteme vielversprechend, weil sie es ermöglichen, die Nutzung eines Produktes zu intensivieren beziehungsweise bis zu einem optimalen Zeitpunkt hin zu verlängern, um so die Ressourcenproduktivität des Gesamtsystems zu erhöhen (Tukker/ Tischner 2006a).

\section{Definitionen: Product-Service Systems}

Mittlerweile ist folgende PSS Definition und Typologie weit verbreitet (Tischner/ Verkuijl/ Tukker 2002):

Ein Product-Service System (PSS) oder Produkt-Dienstleistungssystem ist eine spezifische Art von Wertangebot, das ein Unternehmen (Netzwerk) seinen Kunden anbietet (oder mit ihnen ko-produziert). Ein PSS besteht aus einer Mischung aus materiellen Produkten und immateriellen Dienstleistungen, die so konzipiert und kombiniert sind, dass sie gemeinsam in der Lage sind, die Kundenbedürfnisse zu erfüllen.

Es werden generell drei PSS Kategorien unterschieden (vgl. Abb.1):

(1) produktorientierte PSS, bei denen das Geschäftsmodell noch überwiegend auf den Verkauf von Produkten ausgerichtet ist, aber Dienstleistungen ergänzt werden.

(2) nutzungsorientierte PSS, bei denen das traditionelle Produkt noch eine zentrale Rolle spielt, aber das Geschäftsmodell nicht mehr auf den Verkauf von Produkten ausgerichtet ist. Das Produkt bleibt im Eigentum des Anbieters und wird in einer anderen Form zur Verfügung gestellt und manchmal von einer Reihe von Nutzern gemeinsam genutzt.

(3) ergebnisorientierte PSS, bei denen sich Kunde und Anbieter im Prinzip auf ein zu lieferndes Ergebnis einigen, und es nicht um ein vorher festgelegtes Produkt geht.

Tabelle 2 beschreibt die drei Kategorien mit ihren jeweiligen Unterkategorien. 


\begin{tabular}{|c|c|}
\hline \multirow{2}{*}{$\begin{array}{l}\text { Produktorientierte } \\
\text { PSS }\end{array}$} & $\begin{array}{l}\text { Produktbegleitende Dienstleistung: Der Anbieter verkauft nicht nur ein Produkt, } \\
\text { sondern bietet auch produktbegleitende Dienstleistungen an. Z.B. Wartungs- } \\
\text { vertrag, Finanzierungsmodell, die Lieferung von Verbrauchsmaterialien, oder } \\
\text { eine Rücknahmevereinbarung am Ende der Lebensdauer des Produkts. }\end{array}$ \\
\hline & $\begin{array}{l}\text { Produktbegleitende Beratung/Consulting: Anbieter gibt Ratschläge zur effizien- } \\
\text { testen Nutzung des verkauften Produkts. Z.B. Beratung zur Organisations- } \\
\text { struktur des Teams, das das Produkt einsetzt, die Optimierung der Logistik in } \\
\text { der Produktion etc. }\end{array}$ \\
\hline \multirow{4}{*}{$\begin{array}{l}\text { Nutzungsorientierte } \\
\text { PSS }\end{array}$} & $\begin{array}{l}\text { Produktleasing: Das Produkt wechselt nicht mehr den Eigentümer. Der Anbie- } \\
\text { ter bleibt Eigentümer und ist oft auch für Wartung, Reparatur und Instadn- } \\
\text { haltung verantwortlich. Der Leasingnehmer zahlt eine regelmäßige Gebühr } \\
\text { für die Nutzung des Produkts; hier hat er in der Regel einen unbegrenzten } \\
\text { und individuellen Zugang zum geleasten Produkt. }\end{array}$ \\
\hline & $\begin{array}{l}\text { Produktvermietung oder -sharing: Wie Produktleasing aber der Nutzer hat kei- } \\
\text { nen unbegrenzten und individuellen Zugang; andere können das Produkt zu } \\
\text { anderen Zeitpunkten auch nutzen. Dasselbe Produkt wird nacheinander von } \\
\text { verschiedenen Nutzern verwendet. }\end{array}$ \\
\hline & $\begin{array}{l}\text { Produktpooling: Wie Sharing. Allerdings findet hier eine gleichzeitige Nutzung } \\
\text { des Produkts durch mehrere Nutzer statt. }\end{array}$ \\
\hline & $\begin{array}{l}\text { Pay-per-Service-Einheit: Auch hier kauft der Nutzer das Produkt nicht sondern } \\
\text { zahlt für die Leistung des Produkts entsprechend der Nutzungsintensität. } \\
\text { Z.B. Pay-per-Print-Service von Kopierern oder Pay-per-Wash bei Waschma- } \\
\text { schinen. Der Serviceanbieter übernimmt alle Aktivitäten, die notwendig sind, } \\
\text { um die Nutzung zu gewährleisten. Der Unterschied zur Kategorie Funktiona- } \\
\text { les Ergebnis besteht darin, dass der Nutzer das Gerät noch selbst bedienen } \\
\text { muss. }\end{array}$ \\
\hline \multirow{2}{*}{$\begin{array}{l}\text { Ergebnisorientierte } \\
\text { PSS }\end{array}$} & $\begin{array}{l}\text { Aktivitätsmanagement/Outsourcing: Ein Teil einer Aktivität eines Unterneh- } \\
\text { mens wird an eine dritte Partei ausgelagert. Da die meisten Outsourcing-Ver- } \\
\text { träge Leistungsindikatoren enthalten, um die Qualität der ausgelagerten } \\
\text { Dienstleistung zu kontrollieren, fallen sie in diese Kategorie. Oft ändert sich } \\
\text { die Art und Weise, wie die Tätigkeit ausgeführt wird, kaum. Z.B. Outsourcing } \\
\text { von Catering und Büroreinigung. }\end{array}$ \\
\hline & $\begin{array}{l}\text { Funktionelles Ergebnis: Der Anbieter vereinbart mit dem Kunden die Lieferung } \\
\text { eines Ergebnisses. Er ist im Prinzip völlig frei, wie er das Ergebnis liefert. Z.B. } \\
\text { Unternehmen, die Licht statt Lampen anbieten, die ein bestimmtes „ange- } \\
\text { nehmes Klima“ in Büros, statt Strom oder Klimageräte verkaufen, oder die } \\
\text { Bauern versprechen, Ernteverluste auf ein Mindestmaß zu beschränken, } \\
\text { statt ihnen Pestizide zu verkaufen. }\end{array}$ \\
\hline
\end{tabular}



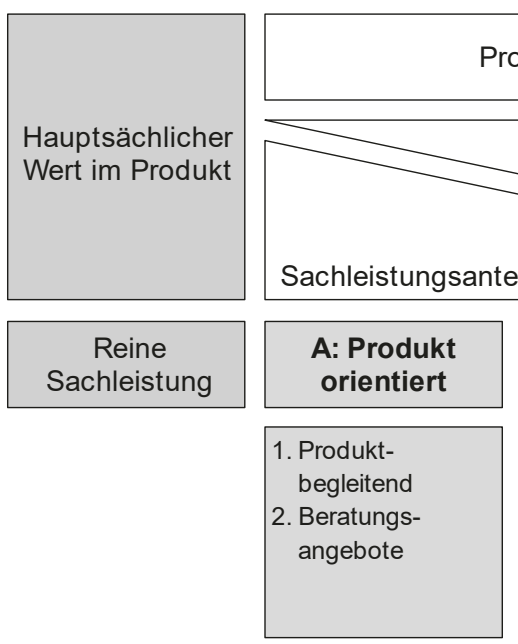

Product-Service-System
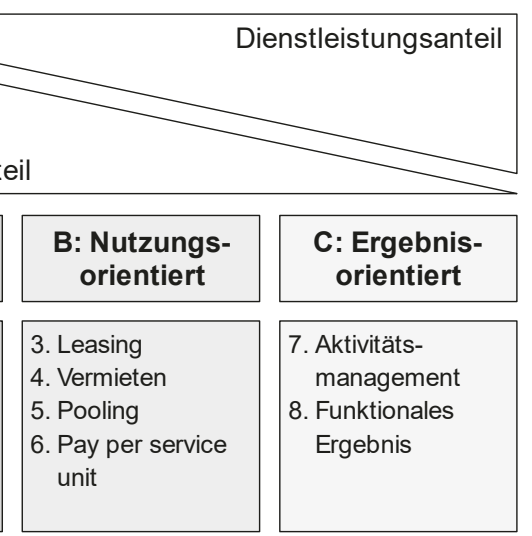
7. Aktivitäts- management
8. Funktionales
Ergebnis

Hauptsächlicher

Wert im Service

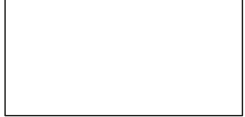

\begin{tabular}{c} 
Reine \\
Dienstleistung \\
\hline
\end{tabular}

Abbildung 1: PSS Kategorien (Quelle: Tischner/ Verkuijl/ Tukker 2002)

Die drei verschiedenen PSS Kategorien haben unterschiedliche Nachhaltigkeitspotenziale, die folgendermaßen beschrieben werden können (Tukker/ Tischner 2006b).

- Produktorientierte Dienstleistungen fügen lediglich Dienstleistungen zu einem bestehenden Produktsystem hinzu. Es können bestenfalls Nachhaltigkeitsverbesserungen von ein paar Dutzend Prozent aufgrund von Aspekten wie besserer Wartung und Lebensdauerverlängerung erzielt werden - höchstens ein Faktor 2 an Ressourceneffizienzsteigerung, wenn die Dienstleistung Reuse, Remanufacturing oder Recycling fördert.

- Nutzungsorientierte Dienstleistungen intensivieren die Nutzung der Produkte. Dies kann zu mittleren (Faktor 2) Verbesserungen führen (vgl. Mont 2004 und Wong 2004).

- Ergebnisorientierte Dienstleistungen sind in der Tat die einzig wahren bedarfsorientierten Dienstleistungen. Unter der Voraussetzung, dass der PSS-Lieferant eine völlig neuartige Art der Funktionserfüllung entwickelt, haben diese ein hohes Potenzial (Faktor X) der Steigerung von Ressourceneffizienz und Reduktion der Umweltbelastungen. 
Tabelle 3: PSS Umweltentlastungspotenziale (Quelle: Tukker/Tischner 2006a)

\begin{tabular}{|c|c|c|c|c|}
\hline \multirow[t]{2}{*}{ PSS Kategorie } & \multicolumn{4}{|c|}{ Umweltbelastung im Vgl. zum Referenzsystem (Produktverkauf) } \\
\hline & Schlechter Gleich & $\begin{array}{l}\text { Kleine } \\
\text { Reduktion } \\
(<20 \%)\end{array}$ & $\begin{array}{l}\text { Deutliche } \\
\text { Reduktion } \\
(<50 \%)\end{array}$ & $\begin{array}{l}\text { Radikale } \\
\text { Reduktion } \\
(<90 \%)\end{array}$ \\
\hline Produktbegleitender Service & \multicolumn{4}{|c|}{ 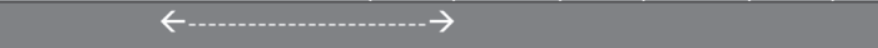 } \\
\hline Produktbegleitende Beratung & \multicolumn{4}{|c|}{$\leftarrow-\cdots$} \\
\hline Leasing & \multicolumn{4}{|c|}{$\leftarrow$} \\
\hline Vermietung und Sharing & \multicolumn{4}{|c|}{ 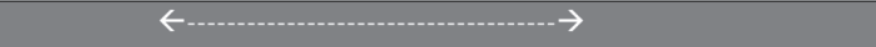 } \\
\hline Pooling & \multicolumn{4}{|c|}{ 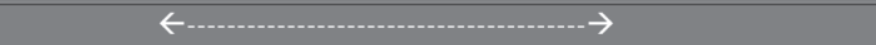 } \\
\hline Pay-per Service Einheit & \multicolumn{4}{|c|}{$\leftarrow----\rightarrow$} \\
\hline Aktivitätsmanagement & \multicolumn{4}{|c|}{ 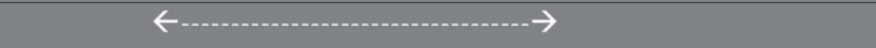 } \\
\hline Funktionelles Ergebnis & \multicolumn{4}{|c|}{ 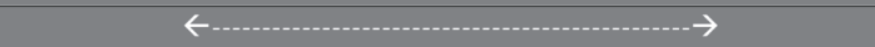 } \\
\hline
\end{tabular}

Die Beurteilung der Nachhaltigkeitspotenziale von PSS bedarf also differenzierter Bewertungsschemata. Die Transformation in Richtung einer nachhaltigen Servicegesellschaft bedeutet einen teils tiefgreifenden Wandel herrschender Nutzungsmuster (Tischner/ Scholl 2004), d.h. Veränderung der Konsumgewohnheiten. Die eigentliche Stärke des PSS-Denkens liegt darin, dass es sich von bestehenden Produktverkaufskonzepten wegbewegt und sich prinzipiell auf den zu erfüllenden Bedarf oder die Funktion konzentriert. Das erhöht die Freiheitsgrade enorm, nachhaltige Verbesserungsmöglichkeiten zu finden. Wenn Nachhaltigkeitsaspekte beim PSS-Design gründlich berücksichtigt werden, können durchaus ungenutzte Potenziale für Nachhaltigkeitsgewinne aufgedeckt werden. Die Nachhaltigkeit muss also in das Produkt-Dienstleistungssystem hinein „designed“ werden.

\section{Wie können Produkt-Dienstleistungssysteme die Kreislaufwirtschaft unterstützen?}

Es gibt viele Parallelen zwischen der Implementierung von Kreislaufwirtschaftsstrategien und Produkt-Dienstleistungssystemen. In beiden Bereichen scheint eine höhere Systemeffizienz in den Re-Think Strategien zu liegen. Bestehende Wertschöpfungsketten durch Materialrecyclingansätze zu ergänzen, ist ein erster Schritt, aber radikalere Verbesserungen können erzielt werden, wenn die gesamte Wertschöpfungskette neu gedacht und organisiert wird inklusive Design der materiellen Komponenten und wo- 
möglich auch neuer Geschäftsmodelle. Beispielsweise steckt im Wandel von produktverkaufs- zu dienstleistungsorientierten Geschäftsmodellen großes Potenzial. Die Einführung von nutzungsorientierten (z.B. Sharing) oder ergebnisorientierten (z.B. Pay-perPerformance) Geschäftsmodellen, sowohl im B2B- als auch im B2C-Sektor, ist ein großer Hebel, um Unternehmen einen Anreiz zu geben, die Lebensdauer der Produkte zu verlängern, diese so intensiv wie möglich zu nutzen, sie so kosten- und materialeffizient wie möglich herzustellen und Teile nach dem Ende der Produktlebensdauer so weit wie möglich wiederzuverwenden. PSS-Strategien können also wichtige Ermöglicher für Kreislaufwirtschaft sein. Tabelle 4 stellt Beispiele von PSS-Strategien zur Förderung der Nachhaltigkeit in jeder der übergeordneten Kreislaufwirtschaftsstrategien dar fokussiert auf eher langlebigere Gebrauchsgüter und das Schließen von technischen Kreisläufen.

Tabelle 4: Kreislaufstrategien und mögliche positive Effekte der PSS Strategien

\begin{tabular}{|c|c|c|c|}
\hline Kreislaufstrategie & Produktorientierte PSS & Nutzungsorientierte PSS & Ergebnisorientierte PSS \\
\hline $\begin{array}{l}\text { Lebensdauerverlän- } \\
\text { gerung durch War- } \\
\text { tung, Reparatur, } \\
\text { Upgrading }\end{array}$ & $\begin{array}{l}\text { Hersteller bietet zusätzlich } \\
\text { zum Produktverkauf War- } \\
\text { tung, Reparatur und Up- } \\
\text { grading Service für seine } \\
\text { Produkte an. }\end{array}$ & $\begin{array}{l}\text { Hersteller/Anbieter nutzt } \\
\text { Produkte so lange wie } \\
\text { möglich und ist selbst für } \\
\text { Wartung, Reparatur und } \\
\text { Upgrading verantwortlich. }\end{array}$ & $\begin{array}{l}\text { Anbieter wählt eingesetzte } \\
\text { Produkte so aus, dass sie } \\
\text { möglichst lange und zuverläs- } \\
\text { sig ihren Dienst tun inklusive } \\
\text { Wartung, Reparatur und Up- } \\
\text { grading. }\end{array}$ \\
\hline Reuse & $\begin{array}{l}\text { Hersteller kauft eigene } \\
\text { Produkte wieder an und } \\
\text { betreibt einen Second } \\
\text { Hand Shop. }\end{array}$ & $\begin{array}{l}\text { Hersteller/Anbieter organi- } \\
\text { siert systematisch Reuse im } \\
\text { eigenen Geschäftsinte- } \\
\text { resse. }\end{array}$ & $\begin{array}{l}\text { Anbieter setzt Produkte so in- } \\
\text { tensiv wie möglich und so } \\
\text { lange wie möglich ein, auch } \\
\text { bei unterschiedlichen Kun- } \\
\text { den. }\end{array}$ \\
\hline Remanufacturing & $\begin{array}{l}\text { Hersteller bietet Produkt- } \\
\text { rücknahme an und führt } \\
\text { Produkte/ Komponenten } \\
\text { wo möglich in die Produk- } \\
\text { tion zurück. }\end{array}$ & $\begin{array}{l}\text { Hersteller/Anbieter nutzt } \\
\text { Komponenten von Ge- } \\
\text { brauchtgeräten intensiv, } \\
\text { um seine Geräte so lange } \\
\text { wie möglich nutzbringend } \\
\text { zu betreiben. }\end{array}$ & $\begin{array}{l}\text { Wenn Anbieter auch Herstel- } \\
\text { ler ist, kann er Komponenten } \\
\text { in der Produktion wieder ein- } \\
\text { setzen, oder er gibt das Pro- } \\
\text { dukt dem Hersteller zurück. }\end{array}$ \\
\hline Recycling & $\begin{array}{l}\text { Hersteller bietet Produkt- } \\
\text { rücknahme an und rezyk- } \\
\text { liert Materialien soweit } \\
\text { möglich und profitabel. }\end{array}$ & $\begin{array}{l}\text { Hersteller/Anbieter ver- } \\
\text { sucht soweit möglich und } \\
\text { profitabel Materialkreis- } \\
\text { läufe zu schließen. }\end{array}$ & $\begin{array}{l}\text { Wenn Anbieter auch Herstel- } \\
\text { ler ist, kann er Materialien in } \\
\text { der Produktion wieder einset- } \\
\text { zen, oder er gibt das Produkt } \\
\text { dem Hersteller zurück. }\end{array}$ \\
\hline
\end{tabular}


Die Bewertung der Nachhaltigkeit von PSS in allen drei Dimensionen (ökologisch, sozial und ökonomisch) und eine genaue Betrachtung des Systems und seiner Rahmenbedingungen ist also unerlässlich, um zu entscheiden, ob ein neues oder verändertes PS System nachhaltiger ist als ein Referenzsystem oder der Zustand vor der Entwicklung des PSS. Zu einer solchen Bewertung gehört zunächst die Definition des zu betrachtenden Systems und seiner Grenzen sowie des Leistungsbündels des PSS ausgehend vom Kundenbedarf. Dann ist es hilfreich zu bestimmen, welcher Kategorie der PSS (s. Abb. 1) die betrachtete Situation zugeordnet werden kann. Schließlich müssen möglichst gute Abschätzungen über materielle und immaterielle Bestandteile des Systems getroffen werden, um zum einen die drei Dimensionen der Nachhaltigkeit zu beurteilen, zum anderen die Marktpotenziale, Nutzerakzeptanz, eventuelle Rebound-Effekte, also ökologisch kontraproduktive Effekte, z.B. durch verändertes Nutzerverhalten, abzuschätzen. Ansätze dazu erläutert der nächste Abschnitt. Schließlich sollte das neue PSS in allen physischen und Service-Elementen so gestaltet werden, dass es möglichst erfolgreich implementiert werden kann und zu möglichst positiven Nachhaltigkeitseffekten führt. In Sinne der Fragestellung hier heißt das auch, dass es die Kreislaufwirtschaft so weit wie möglich fördert.

\section{Methoden und Werkzeuge für Product-Service System Design}

Es existieren mittlerweile zahlreiche Methoden und Werkzeuge für die Gestaltung von Produkt-Dienstleistungssystemen, die aus der Service Design Domäne, aus dem Produkt Design oder aus der Nachhaltigkeits-Design Domäne stammen. Vorgeschlagene Prozesse des PSS Design beinhalten üblicherweise folgende Schritte (vgl. Tukker/ Tischner 2006a):

\section{Schritt 1: Analysieren}

- der aktuellen Situation

- des Referenzprodukts/der Referenzdienstleistung

- der Kundenbedürfnisse und -erwartungen

- der internen Situation der Unternehmen und ihrer externen (potenziellen) Partner und damit die Erkundung und Identifizierung neuer Geschäftsmöglichkeiten im PSS-Bereich. 


\section{Schritt 2: Erstellen und Detaillieren neuer Ideen}

- basierend auf den Erkenntnissen bzw. dem vorhandenen Wissen über Geschäftsmöglichkeiten werden neue Ideen für PSS generiert,

- die vielversprechendsten werden ausgewählt,

- die ausgewählte(n) Idee(n) wird/werden detailliert,

— abschließende Bewertung zeigt, ob das detaillierte Konzept gut genug ist, um realisiert zu werden.

\section{Schritt 3: Realisierung des detaillierten Konzepts}

- Vorbereitung der Markteinführung, Entwicklung einer Marketingstrategie

- Produktion der materiellen und immateriellen Teile des PSS

- Markttest

- Markteinführung

- Beobachtung und Bewertung des Markterfolgs

— Überprüfung/Überarbeitung des PSS-Entwicklungsprozesses

Für jeden dieser Schritte stehen wiederum zahlreiche Werkzeuge zur Verfügung, von der Nachhaltigkeits-Evaluierung und dem Mapping des bestehenden Referenzsystems, über die Ideenentwicklung für neue Produkt-Dienstleistungssysteme, dem Entwerfen von Akteurskonstellationen und Analysieren der Kundennachfrage oder der Motivation der Stakeholder bis hin zur Geschäftsmodellentwicklung und ökologischen-ökonomischen Bewertung des neuen PSS etc. Im Unterschied zur klassischen Produktentwicklung braucht es für ein neues PSS in der Regel Kooperation von weiteren Akteuren, und die Kombination von materiellen Produktbestandteilen mit eher immateriellen Service-Bestandteilen. Oft ändern sich die materiellen Ströme im PS System, das bedarf einer neuen Logistik. Außerdem basieren solche Systeme meistens auf veränderten Informationsflüssen und gesteigerter Digitalisierung ebenso wie geänderten Finanzströmen und Aufteilung des Einkommens auf mehrere Akteure usw. Optimal ist es, wenn die Entwicklung/Anpassung der materiellen Bestandteile des Systems (Produkte) parallel und im engen Austausch mit der Entwicklung der Serviceanteile geschieht.

Eine größere Herausforderung stellt die Nachhaltigkeitsbewertung von PSS dar. Übliche Produktbewertungstools (LCA/Ökobilanz, lebenszyklusweite Bewertungschecklis- 
ten) sind ohnehin schon anspruchsvoll jedoch oft nicht in der Lage, mit der Komplexität und den Unsicherheiten eines PS Systems umzugehen. Um dennoch richtungssichere Abschätzungen der Nachhaltigkeit von neuen PSS im Vergleich zu einem Referenzsystem vornehmen zu können, wurden qualitativ bewertende Checklisten entwickelt, wie z. B. die System SWOT Analyse oder das folgende PSS Screeningtool aus dem SusProNet Projekt (Tukker/Tischner 2006a).

Ein weiteres hilfreiches Tool ist die System Map, zur Visualisierung von bestehendem und neuem System (vgl. Manzini/ Collina/ Evans 2004). Hier werden die Kern- und erweiterten Akteure eingetragen, die Aktivitäten, aber auch Material-, Finanz- und Informationsströme durch das System. Gleichzeitig können „Pain Points“ und „Windows of Opportunities" identifiziert werden (vgl. Abb. 2).

Die Entwicklung neuer PSS Geschäftsmodelle unterstützen z. B. der Business Model Navigator der Universität St. Gallen (Gassmann/ Frankenberger/Csik 2013) und das Business Model Canvas (vgl. Osterwalder 2004).

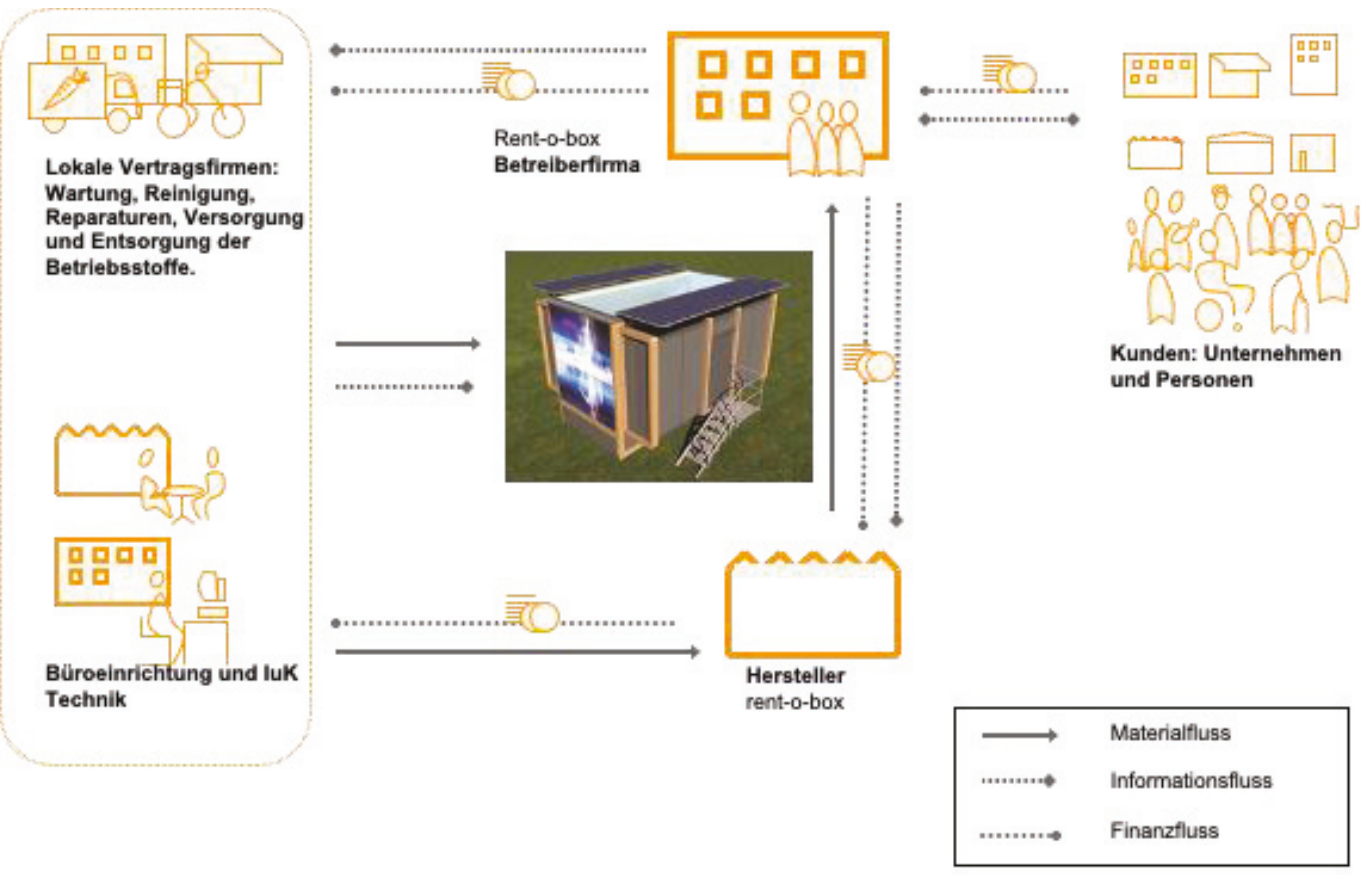

Abbildung 2: Vereinfachte System Map für das Rent-o Box Konzept von econcept 
Da in Business to Consumer PSS ein Erfolgshemmnis oft darin liegt, dass Konsumenten Produkte immer noch lieber als ihr Eigentum erwerben, anstatt sie zu mieten oder zu sharen, kann hier ein sanftes „Nudging” hilfreich sein, also das Design des PSS mit besonders guten Anreizen und überzeugenden Argumenten für Konsumenten. Hierbei hilft die "Design with Intent" (Design mit Absicht) Methode (vgl. Lockton/Harrison/Stanton 2010 und http://designwithintent.co.uk). Durch sie werden die unterschiedlichsten Wege aufgezeigt, von autoritär bis spielerisch, wie Nutzerverhalten durch Designinterventionen beeinflusst werden kann.

Tabelle 5: Nachhaltigkeitsscreening von neuen PSS im Vergleich zum Referenzsystem

\section{A) Ökonomische Aspekte $\quad$ Score}

$(1=$ besser, $0=$ gleich, -1 = schlech -

ter)

- Wie profitabel/wertvoll ist die Lösung für die Anbieter? (Das kann ein Konsortium von Unternehmen sein) Einschließlich Produktionskosten, Kapitalkosten und Marktwert der Lösung für den/die Anbieter? Ist sie günstiger zu produzieren als das Konkurrenzprodukt?

- Wie profitabel/wertvoll ist die Lösung für Kunden/Verbraucher? Gibt es eine konkrete, greifbare Einsparung an Zeit, Materialeinsatz etc. für den Kunden? Bietet sie einen „unbezahlbaren“, immateriellen Mehrwert wie Wertschätzung, Erlebnisse usw., für den Kunden bereit sind, mehr zu bezahlen? (beides im Vergleich zum traditionellen Produktsystem)

- Wie schwierig zu implementieren und riskant ist die Lösung für die Anbieter? Kann ein versprochenes Ergebnis mit hoher Wahrscheinlichkeit gemessen und geliefert werden, oder hat der Kunde einen hohen und unkontrollierbaren Einfluss auf die Kosten? Wann wird der Return on Investment erwartet?

- Inwieweit trägt die Lösung zur Fähigkeit bei, die Wertschöpfung in der Zukunft zu erhalten? Verschafft sie dem Konsortium, welches das PSS jetzt und in Zukunft auf den Markt bringt, eine entscheidende und dominante Position in der Wertschöpfungskette? 


\section{B) Umweltaspekte}

\section{Score}

$(1$ = besser, $0=$ gleich, -1 = schlech-

ter)

- Wie gut ist die Lösung in Bezug auf die Materialeffizienz (einschließlich Inputs und Outputs, Abfall, Kreislaufwirtschaft)

- $\quad$ Wie gut ist die Lösung in Bezug auf die Energieeffizienz (Energieeinsatz und Rückgewinnung von Energie, ohne Transport)

- Wie gut ist die Lösung in Bezug auf Toxizität (einschließlich Input/Output von Gefahrstoffen und Emissionen, ohne Transport)

- Wie gut ist die Lösung in Bezug auf die Transporteffizienz (Transport von Gütern und Personen einschließlich Transportentfernungen, Transportmittel, Volumen und Verpackung)

SUMME

\section{C) Soziale Aspekte}

\section{Score}

$(1$ = besser, $0=$ gleich, -1 = schlechter)

- $\quad$ Trägt das PSS zur Anzahl und Qualität der Arbeitsplätze in der Produktionskette bei (Umwelt, Gesundheit, Sicherheit; Bereicherung des Lebens der Arbeitenden durch Bildungsmöglichkeiten usw.)

- Trägt das PSS zur „Bereicherung" des Lebens der Nutzer bei (soziale Aspekte in der Wertschöpfungskette, Auswirkungen auf die Gesellschaft, indem es Lernmöglichkeiten bietet, Aktion statt Passivität ermöglicht, Kompetenzen fördert usw.)

- $\quad$ Trägt das PSS zur Gerechtigkeit innerhalb und zwischen den Generationen bei (Wohlstands- und Machtverteilung zwischen gesellschaftlichen Gruppen, industrialisierten und weniger industrialisierten Ländern, keine Verschiebung von Problemen auf die nächste Generation usw.)

- Inwieweit trägt die Lösung zur Achtung kultureller Werte und kultureller Vielfalt bei, z.B. maßgeschneiderte Lösungen, Beitrag zum sozialen Wohlergehen von Gemeinden, Regionen usw. (kulturelle Werte)

SUMME 


\section{Fallbeispiele: Rent-O-Box und Kreislauf der Abfallbehälter}

Abschließend sollen zwei Fallbeispiele das Potenzial von PSS zur Unterstützung von Kreislaufwirtschaftsstrategien verdeutlichen.

\section{Rent-O-Box: autarkes, mobiles Mietbüro}

Büroarbeit der Zukunft folgt dem Trend der Verlagerung vom stationären Büro zur eher virtuellen, eher ortsunabhängigen und eher kreativen Teamarbeit - und das nicht erst seit der Corona Pandemie. Für moderne Büro Nomaden entwickelte econcept eine Sharing-Büro-Zelle: ein unabhängiges, mobiles "tiny office“, das sich an den unterschiedlichsten Orten aufstellen lässt und eine komplette Büroinfrastruktur mit modernster dematerialisierter Technik zur Verfügung stellt. Nutzer:innen buchen die Zellen bei einer Betreibergesellschaft (ähnlich Carsharing). Der Betreiber organisiert neben der Buchung auch den Unterhalt (Aufstellung, Reinigung, Reparatur) und die Versorgung (Betriebsmittel, Getränke, Snacks). Neben der Arbeitsinfrastruktur enthält die Rent-O-Box auch eine Erholungs- und Entspannungszone. Die kleinen Büroeinheiten werden in regelmäßigen Abständen gewartet, repariert und, wenn sie einmal als Büros ausgedient haben, einer weiteren Verwendung zugeführt: vom Gartenhaus bis zur kurzzeitige Unterbringung von Geflüchteten gibt es hier vielfältige Szenarien. Die Nachhaltigkeit dieses PSS liegt einerseits in der Idee an sich: der Flächenbedarf an stationären Bürogebäuden wird reduziert, die Raumnutzung ist durch das Mietverfahren sehr effizient und Pendlerverkehre werden durch die Möglichkeit dezentral zu arbeiten verringert. Gleichzeitig erfolgt die Konstruktion und die Auswahl der Materialien und technischen Einrichtungen nach ökologischen Kriterien: Langlebigkeit, Energieverbrauch, Kreislauffähigkeit, etc. Das kleine Gebäude sammelt Regenwasser für die eingebaute Mini-Sanitärzelle und erzeugt Strom per Photovoltaikpanels. So ermöglicht sie über einen längeren Zeitraum eine autarke Nutzung unabhängig von Strom- und Wasseranschluss.

\section{Der geschlossene Kreislauf der Abfallcontainer}

Ein europäischer Hersteller von Abfallcontainern für Entsorgungsbetriebe, Städte und Kommunen hat einen geschlossenen Kreislauf für seine Abfallbehälter aufgebaut. Das Unternehmen schließt die Materialkreisläufe, indem es einen Abholservice für kaputte Abfallbehälter anbietet, also eigene und auch fremde Abfallbehälter bei Entsorgungsbetrieben, Städten und Kommunen abholt. Die Tonnen werden gleich im Fahrzeug zerlegt, das Kunststoffmaterial (HDPE) wird regranuliert, gesäubert und anschließend in der Produktion wieder zu neuen Mülltonnen verarbeitet. Auch die Achsen und Räder werden wo möglich wiederverwendet. So werden Abfallaufkommen und Einsatz von 
neuen Rohstoffen drastisch reduziert und die Materialkosten für das produzierende Unternehmen vermindert. Das Farbkonzept von Tonnen und Deckeln wurde dementsprechend angepasst. Da das Unternehmen die Abfallbehälter auch im Mietservice anbietet, reduzieren sich die Anschaffungskosten für die Kommunen bzw. Entsorgungsbetriebe und erhöht sich die Kundenbindung. So konnte eine win-win-win Situation erzeugt werden.
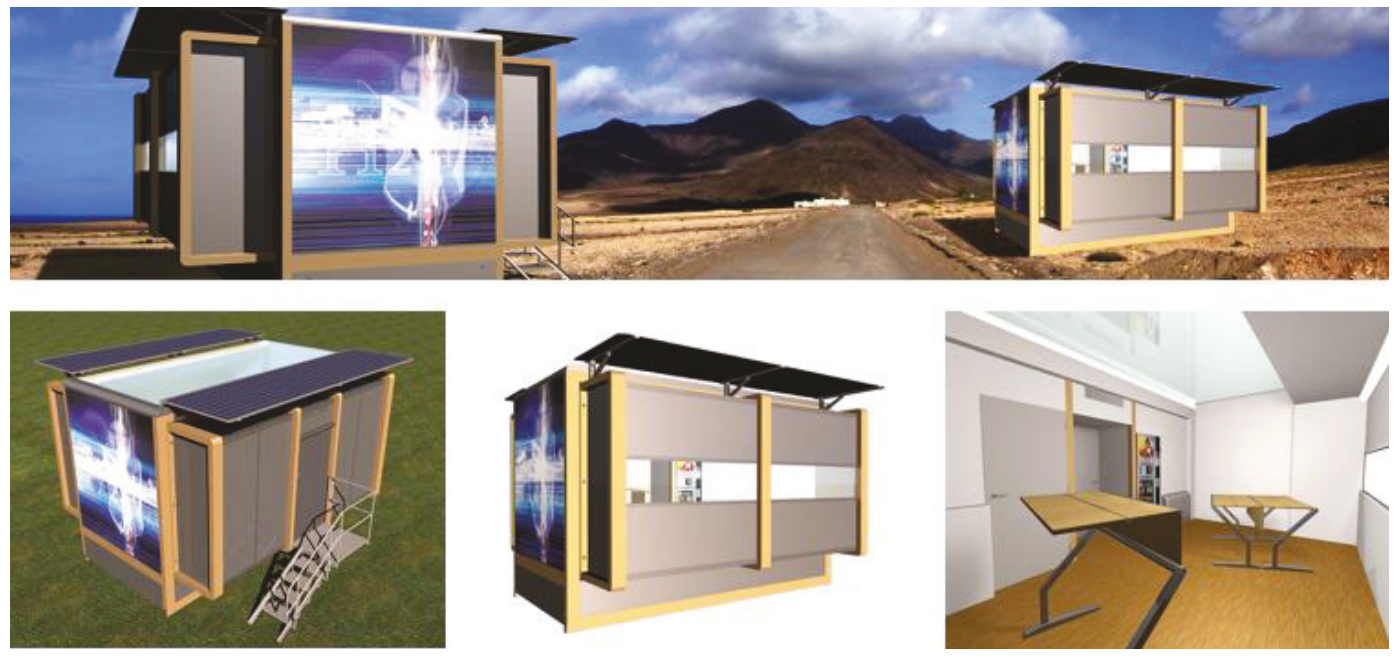

Abbildung 3: Ansichten der Rent-O-Box. Quelle: econcept

\section{Fazit}

Circular Economy/Kreislaufwirtschaft und Circular Design/Kreislaufdesign sind vielversprechende Strategien zur Reduktion von Ressourcenverbrauch und Klimawandel. Jedoch greift es zu kurz, vom Abfall her zu denken. Effizienter und effektiver sind i.d.R. Kreislaufwirtschaftsstrategien, die von Anfang an in Produktentwicklung und Design eingeplant und die unter Einbezug von alternativen Geschäftsmodellen entwickelt wurden (Nutzen statt Eigentum, PaaS, Pay per Use, Sharing etc.). Immer dann, wenn der Produzent/Anbieter die Produkt nicht verkauft, sondern die Nutzung oder ein Endergebnis anbietet, steigen die Anreize für inn die Produkte möglichst lange und möglichst effizient zu nutzen. Der Serviceanteil wird wichtiger im Geschäftsmodell. In vielen Fällen kann durch Einführung solcher Produkt-Dienstleistungssysteme (Product-Service Systems) die Ressourcen- und Energie-Effizienz im System gesteigert werden im Vergleich zu üblichen Produktverkaufslösungen. Rebound Effekte können verhindert 
werden. Solche Product-Service Systeme sind aber nicht automatisch nachhaltig, sondern die Nachhaltigkeit muss sorgfältig in das System hinein gestaltet werden. Dazu sollten PSS Designer eine Analyse und Bewertung des bestehende Systems vornehmen inklusive der involvierten Akteure und der Ressourcen-, Energie-, Informationsund Geldflüssen durch das System. Die Stärken und Schwächen des bestehenden Systems sollten identifiziert und dann neue PS Systeme entwickelt werden, die Schwächen und Probleme eliminieren, Wertschöpfung für möglichst viele Akteure steigern und gleichzeitig Ressourcen- und Energieverbräuche im Gesamtsystem verringern, die Kreislaufführung von Produkten, Komponenten und Materialien steigern. Das neue bzw. verbesserte System kann dann wiederum skizziert detailliert und bewertet werden. Hilfreiche Methoden dazu, sind System SWOT Analyse, das PSS Screening Tool, System Map mit Pain Points und Windows of Opportunities, Business Model Navigator und Business Model Canvas. So kann das Einführen von mehr und mehr Serviceanteilen in Geschäftsmodelle und das Fokussieren auf das Anbieten von Nutzen (statt auf ein zu verkaufendes Produkt) dazu führen, dass Kreislaufwirtschaftsstrategien stärker im Interesse von Anbietern und Nutzer:innen liegen.

\section{Literaturverzeichnis}

acatech, Circular Economy Initiative Deutschland, SYSTEMIQ (Eds.) (2020): Circular Business Models - Overcoming Barriers unleashing potentials Executive Summary and Recommendation, Munich/London

Braungart, M., McDonough, W. (2014): Cradle to Cradle: Einfach intelligent produzieren, Piper Taschenbuch; 7. Edition (17. Februar 2014), München

Earth Overshoot Day: Country Overshoot Days, 2020. URL: https://www.overshootday.org/newsroom/country-overshootdays/ abgerufen 02.12.2020

Europäische Kommission (2019): Der europäische Grüne Deal, Brüssel

Europäische Kommission (2020): A new Circular Economy Action Plan. For a Cleaner and more Competitive Europe, 2020. https://eur-lex.europa.eu/resource.htm|?uri=cellar:9903b325-6388-11ea-b735-01aa75ed71a1.0017.02/DOC_18format=PDF, abgerufen 11.06 .2020

Gassmann, O., Frankenberger, K. \& Csik, M. (2013): Geschäftsmodelle entwickeln. 55 innovative Konzepte mit dem St. Galler Business Model Navigator. Hanser Verlag

International Resource Panel: Global Resources Outlook. 2019: Natural Resources for the Future We Want, 2019. https://www.resourcepanel.org/reports/global-resources-outlook, abgerufen 22.06.2020

International Resource Panel (2020): Resource Efficiency and Climate Change, Nairobi, Kenya

Kreislaufwirtschaftsgesetz vom 24. Februar 2012 (BGBI. I S. 212), das zuletzt durch Artikel 2 Absatz 2 des Gesetzes vom 9. Dezember 2020 (BGBI. I S. 2873) geändert worden ist. https://www.gesetze-im-internet.de/krwg/KrWG.pdf abgerufen 27.03.2021 
Lockton, D., Harrison, D.J. \& N.A. Stanton (2010): Design with Intent: 101 Patterns for Influencing Behaviour Through Design v.1.0, Windsor: Equifine 2010

Lund, R. T. (1977): Making Products Live Longer, in: Technology Review, No. 1, pp. 49-55

Manzini, E., Collina I. \& S. Evans (2004): Solution oriented partnership, how to design industrialised sustainable solutions. Cranfield University. Cranfield, UK

Material Economics (2018): The Circular Economy - a Powerful Force for Climate Mitigation. https://materialeconomics.com/publications/the-circular-economy-a-powerful-force-for-climate-mitigation-1 abgerufen 02.12.2020

Mont, 0. (2004): Product-service systems: Panacea or myth? Ph.D. Thesis, IIIEE, Lund University, Sweden

OECD [Organisation for Economic Co-operation and Development] (Ed.) (1982): Product Durability and Product Life Extension, Paris

Osterwalder, A. (2004): The Business Model Ontology: A Proposition in a Design Science Approach, Dissertation, University of Lausanne

Schmidt-Bleek, F., Tischner, U. (1995): Produktentwicklung, Nutzen gestalten - Natur schonen, Wirtschaftskammer Österreich (Ed.), WIFI

Stahel, W. (1991): Vertiefungsstudie zu Langlebigkeit und Materialrecycling im Bereich der Produkte, Stuttgart

Stahel, W. (2010): The Performance Economy, Second Edition, Palgrave-MacMillan, London, (first edition 2006)

Tischner, U., Verkuijl, M., \& A. Tukker (2002): First Draft PSS Review. SusProNet Report, draft 15. December 2002. Available from econcept, Pulheim, Germany; TNO-STB, Delft, The Netherlands

Tischner, U. \& G. Scholl (2004): Nachhaltige Produkt-Dienstleistungssysteme, in: Umwelt-Wirtschafts-Forum, Ausgabe $1 / 2004$

Tischner, U., Ryan, C. \& C, Vezzoli (2009): Chapter 7: Product-Service-Systems, in: Design for Sustainability, A step-by-step approach, United Nations Environment Program (Ed.), Paris

Tukker, A. \& U. Tischner (2006a): New Business for Old Europe, Product-Service Development, Competitiveness and Sustainability, Greenleaf Publishing, Sheffield UK

Tukker, A. \& U. Tischner (2006b): Note from the field. Product-services as a research field: past, present and future. Reflections from a decade of research. in: Journal of Cleaner Production, Volume 14, Issue 17, 2006, Pages 1552-1556

US Congress (1976): Resource Conservation and Recovery Act of 1976, Public Law 94-580 94th Congress, USA

Van Halen, C., Vezzoli, C., \& Wimmer R. (2005) Methodology for Product-Service System Innovation, How to implement clean, clever and competitive strategies in European industries, Royal Van Gorcum, Assen, Netherlands

VDI-Fachbereich Produktentwicklung und Mechatronik (2002): VDI 2243 - Recyclingorientierte Produktentwicklung, Hrsg. VDI-Gesellschaft Produkt- und Prozessgestaltung (ursprünglicher Titel von 1991: Konstruieren recyclinggerechter technischer Produkte)

Weber, T. \& M. Stuchtey (2019): Deutschland auf dem Weg zur Circular Economy - Erkenntnisse aus europäischen Strategien (Vorstudie), München 
Wong, M. (2004): Implementation of innovative Product Service Systems in the Consumer Goods Industry. dissertation thesis, University of Cambridge, department of Engineering, UK

\section{Kontakt}

Dipl.-Des. Ursula Tischner, MFA B. Sc. CEO, econcept - Agentur für nachhaltiges Design Albatrosweg 11

50259 Pulheim

www.econcept.org 